\title{
Generating, solving and the mathematics of Homo Sapiens. Emil Post's views on computation
}

\author{
Liesbeth De Mol* \\ elizabeth.demol@ugent.be
}

\begin{abstract}
"For if symbolic logic has failed to give wings to mathematicians this study of symbolic logic opens up a new field concerned with the fundamental limitations of mathematics, more precisely the mathematics of Homo Sapiens."
\end{abstract}

Emil Leon Post, 1936

\section{Introduction}

What is a computation? What does it mean to compute something and in how far does it make sense to talk about computation outside of mathematics? These fundamental questions have not received a satisfactory answer yet, despite the well-known "Church-Turing thesis". Roughly put, the Church-Turing thesis states that the set of the "computable" - in the vague, intuitive sense - coincides with the Turing computable, hence rendering a vague notion exact and thus, if accepted, setting a borderline between the computable and the non-computable. However, knowing/assuming that the "computable" is that which can be computed by a Turing machine (or any other formal device that is logically equivalent to it) does not necessarily imply that one knows if and how one can compute whether or not it will rain in Paris on June 23, 2112 or what will be the next sentence of this paper. Viz., to accept that the "computable" is the Turing computable, does not necessarily imply that one knows the universe of the computable.

Since 1936, the year Church and Turing each proposed their theses, these questions have only gained in significance, perhaps not so much because of advancements in mathematical logic, but rather due to the development of what is often understood as the physical realization of computability - the digital, electronic stored-program and general-purpose computer. It was and is this

*This research was supported by the Fund for Scientific Research, Flanders, Belgium. I would like to thank the people at the Manuscripts Department, American Philosophical Society for their assistance with the Emil Leon Post papers as well as the people at the Department of Rare Books and Special Collections, Princeton University Library for their assistance with the Alonzo Church papers. I am also indebted to Maurice Margenstern for some very interesting discussions on (the nature of) computation (in nature) especially on the significance of tag systems and research on the boundaries of decidability and undecidability. 
machine that has effectively widened the scope of the computable and brought computations to people who are and were not really interested in the problems of mathematical logic. It is this machine which has made it possible to physically implement a wide range of computational processes, problems and objects and it was hence the computer that made it relevant to non-logicians to broaden and study the field of the computable and the non-computable, making it necessary to pose questions like: what could be a high-quality weather prediction software? It is exactly this extension of the scope of the computable that explains why it is no longer considered "heretical" ${ }^{1}$ to say that all processes in "nature" going from the human mind to the growth of plants - can be understood as computations, or, much stronger, that everything in "nature" can be computed by a Turing machine.

Despite or perhaps due to the fact that the scope of the computable has effectively been extended beyond the field of mathematical logic, the ChurchTuring thesis, as a philosophical thesis but also as a mathematical definition, is still the uncontested delimiter of the computable. It is here that lies part of its beauty, viz., its two-facedness of opening up the field of computation and, at the same time, imposing a fundamental limitation on it and hence also setting the stage for the non-computable. It is this 'Janus face' of the Church-Turing thesis, going to the core of the thesis, that is sometimes too much underestimated in current debates on the Church-Turing thesis.

To celebrate and honor Turing in 2012 is also to celebrate the Church-Turing thesis. However, to celebrate this achievement is also to acknowledge its historical, philosophical and mathematical richness. Even though one must and should fully acknowledge Turing's fundamental contributions, the fact that hardly any other historical sources are examined in some of the current discussions, including those that allegedly claim to give a "historical" account, can only result in views that are incapable of acknowledging exactly this richness. This is what I would like to do here by exposing the reader to Emil Leon Post's thoughts and results on the 'Church-Turing thesis'. It was Post, who besides Church and Turing, also proposed a "thesis" in 1936. It was Post who had already developed a model of computability that is very different from Turing's in 1921, and, it was also Post who, unlike Church and Turing, insisted on the view that Church's and Turing's thesis should be regarded as a hypothesis, and, ultimately, a natural law rather than as a mathematical definition.

\section{Why Turing rules}

In 1936 both Church and Turing published their celebrated theses. They proposed their respective theses as formal definitions of an otherwise vague and intuitive notion. It were these definitions that allowed Church and Turing to tackle the famous Entscheidungsproblem for first-order logic. Indeed, in order to prove the incomputability of this problem, it was necessary to have a formal

\footnotetext{
${ }^{1}$ See Alan Turing's [27] where he describes the idea of intelligent machinery as heretical.
} 
definition of computability. In his definition, Church identified the vague notion of an effectively calculable function of positive integers with the formal notions of general recursive functions (or, equivalently, $\lambda$-definable function) [1]. Turing identified the vague notion of computable numbers, i.e., "the real numbers whose expressions as a decimal are calculable by finite means" [26, 230], with those numbers computable by a Turing machine .

Even though in the literature Church's thesis and Turing's thesis are mostly not differentiated but put together under the header "Church-Turing thesis" because of their logical equivalence, it is Turing's that is usually considered as being superior to Church's thesis. To put it in Church's words: "[Turing's thesis makes] the identification with effectiveness in the ordinary (not explicitly defined) sense evident immediately" [2] Gödel also shared this view and judged that with Turing's work one has "for the first time succeeded in giving an absolute definition of an interesting epistemological notion" [8]. Soare recently even went a step further by stating that [i]t was Turing alone who [...] gave the first convincing formal definition of a computable function" [25].

But why exactly is Turing's thesis so much more appealing than Church's? This issue has already been discussed in detail in [7, 23, 24] and comes down to the fact that unlike Church, Turing's thesis makes a "direct appeal to intuition" $[26,249]$. Church only arrived at his thesis after several research results on $\lambda$-calculus had been established. It was its equivalence with general recursive functions and the fact that Church, Kleene and Rosser were able to $\lambda$-define any function they could come up with, that resulted in Church's formulation of the thesis, first in terms of $\lambda$-calculus and later in terms of general recursiveness. In other words, Church did not start out from the idea of trying to formally capture some intuition, but, on the contrary, only saw that the formalism he was working with might be powerful enough to capture all "calculable" functions after a thorough analysis of that formalism. This explains why defining effective calculability in terms of $\lambda$-definability or general recursiveness is not "intuitively appealing" since neither of these symbolic systems was constructed with that purpose in mind.

In contrast, Turing $d i d$ start out from the intuitive and vague idea of a man in the process of computing a number, and tried to find a formalism that somehow captures the essential features of any such process. As Turing formulated it [26, 249]:

The real question at issue is: "What are the possible processes which can be carried out in computing a number?"

The formal notion of a Turing machine resulted from an analysis of the human processes involved in computing a number by extracting the essential features of such processes, and introducing them as conditions, like, for instance, the idea that the number of symbols the "computor" (the human computer) can write down is finite. These conditions almost naturally lead to the concept of a Turing machine (See the work of Sieg and Gandy [7, 23] for more details). Thus, unlike Church, Turing started out from a "real-world" situation and derived an 
idealized machine out of it. In this sense, Turing's thesis does aim at establishing a relation between something physical - the (human) processes involved in computing a number - and something symbolic - the Turing machine.

That Turing's thesis is considered as the better thesis is thus explained by the fact that Turing tackled the problem from the direction of finding a good formalization of a vague idea, whereas Church tackled the problem from the other side. ${ }^{2}$

What is mostly forgotten or at least underestimated in this context is that Emil Leon Post had formulated two different theses, one in '21 and one in '36, as a result of tackling similar problems from both directions. He formulated his first thesis after having realized that the formal devices he was studying are much more powerful than he had expected. Being unsatisfied with this thesis for the same reasons as Gödel was with Church's, he then formulated a second thesis which was published in 1936 and involves a formal device which is almost identical to the Turing machine. It resulted from an analysis of "all the possible ways in which the human mind [can] set up finite processes" [21, 387] to "solve" a problem.

\section{Two Theses, two sides}

Emil Post had two very different though logically equivalent theses. The first was formulated in 1921 and was the necessary epistemological assumption for Post's "proof" that there exist absolutely unsolvable problems, like for instance the famous halting problem. ${ }^{3}$ Here are two different formulations of Post's thesis I, first identified as such by Martin Davis [5]:

Post's Thesis I. (simple) For every set of sequences for which a process can be set-up to generate it there is also a normal system which will generate it.

Post's Thesis I. (complete) Every generated set of sequences on a given set of letters $a_{1}, a_{2}, \ldots, a_{\mu}$ is a subset of the set of assertions of a system in normal form with primitive letters $a_{1}, a_{2}, \ldots, a_{\mu}, a_{1}^{\prime}, a_{2}^{\prime}, \ldots, a_{\nu}^{\prime}$, i.e., the subset consisting of those assertions of the normal system involving the letters $a_{1}, a_{2}, \ldots, a_{\mu}$.

It would take another 15 years before Post proposed a second thesis, which was published in his short note Finite combinatory processes - Formulation I [19]. This note was a reaction on Church's thesis which Post found unsatisfactory. This second thesis uses a formalism which is almost identical to the Turing

\footnotetext{
${ }^{2}$ Note that one of Church's main arguments in support of his thesis [1] is also circular and hence contains a fundamental flaw (See $[7,23,24]$ ).

${ }^{3}$ These results were not published in 1921 . Post waited about 20 years before he submitted the manuscript Absolutely unsolvable problems and relatively undecidable propositions Account of an anticipation to the American Journal for Mathematics which contained a description of his research during the period 1920-21. It was rejected by Hermann Weyl. Post's letter of submission and part of Weyl's rejection letter are printed in [6]. Only a significantly abbreviated version of it was finally published as Post's influential [20]. It would take about another 20 years before Martin Davis posthumously published the manuscript in his [4].
} 
machine. It is in this sense that Post's 1936 contribution "contains the same idea as Turing's paper" [10,61]. Here is Post's thesis II in its simple and more technical form:

Post's Thesis II. (simple) Solvability of a problem in the intuitive sense coincides with solvability by formulation 1

Post's Thesis II. (complete) Any decision problem is solvable in the intuitive sense if it can be stated as a 1-given problem for which one can set-up a finite 1-process which results in a 1-solution to the problem.

Several question can and should be posed here: where do these two theses come from? What kind of logical and philosophical analysis and views underlie these two different theses? Why did Post find it necessary to propose a second thesis and, above all, what kind of insights can we gain nowadays from these two theses? We need to dig a bit deeper here into Post's work to tackle these questions.

\subsection{Post's thesis I: Generating sequences and limits of the computable}

In his $\mathrm{PhD}$ Introduction to a general theory of elementary propositions, published in 1921 [17], Post introduces the idea to develop the most general form of logic, and, since he considered logic as the foundation of mathematics, ultimately mathematics. Such general forms he could then use as "instruments of generalization" [17, 173] which make possible a study of the general properties and problems of the whole of mathematics. It was Post's view that if one wants to study the general properties of logic and mathematics then one needs not one particular system of symbolic logic or mathematics, but a general form that comprises all such possible systems. Given such a form he believed it possible to generalize some of the fundamental results from his $\mathrm{PhD}$ - the completeness, consistency and decidability of propositional logic - to the whole of mathematics. This ambitious "programme" is announced in the introduction to his $\mathrm{PhD}$ $[17,164]$.

After his $\mathrm{PhD}$ was finished Post became a Proctor fellow in Princeton during the period 1920-21. It was during that time that he set himself the goal of proving the decidability of the decision problem for first-order $\operatorname{logic}^{4}$ which Post called a finiteness problem, and, ultimately, for the whole of mathematics. He "was proposing no less than to find a single algorithm for all of mathematics" [6]. His approach to this problem? The development of a general form characterized by the method of "combinatory iteration" which "eschews all interpretation" [21, 386]. More specifically [18]:

[T] he method of combinatory iteration completely neglects [...] meaning, and considers the entire system purely from the symbolic stand-

point as one in which both the enunciations and assertions are groups

\footnotetext{
${ }^{4}$ The famous Entscheidungsproblem proven incomputable by Church and Turing.
} 
of symbols or symbol-complexes [....] and where these symbol assertions are obtained by starting with certain initial assertions and repeatedly applying certain rules for obtaining new symbol-assertions from old.

So, what kind of form would one expect to result from such radical formalism? In his PhD Post had in fact already formulated such a form initially called the generalization by postulation and later the canonical form A. However, this did not immediately lead to the desired ultimate algorithm and Post started to develop other forms and study problems related to this finiteness problem. After some months this resulted in Post's 'frustrating' tag systems.

Definition 1. (v-tag system) A tag system $T$ consists of a finite alphabet $\Sigma$ of $\mu$ symbols, a deletion number $v \in \mathbb{N}$ and a finite set of $\mu$ words $w_{0}, w_{1}, \ldots, w_{\mu-1} \in \Sigma^{*}$ called the appendants, where any appendant $w_{i}$ corresponds to $a_{i} \in \Sigma$. A v-tag system has a deletion number $v$.

In a computation step of a tag system $T$ on a word $A \in \Sigma^{*}, T$ appends the appendant associated with the leftmost letter of $A$ at the end of $A$, and deletes the first $v$ symbols of $A$. This computational process is iterated until the tag system produces the empty word $\epsilon$ and hence halts. To give an example, let us consider the one tag system mentioned by Post [20,21] with $v=3,0 \rightarrow 00$, $1 \rightarrow 1101[20,21]$. If the initial word $A_{0}=110111010000$ we get the following productions:

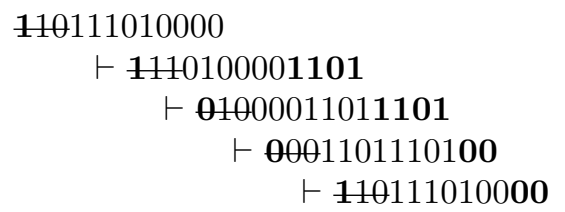

The word $A_{0}$ is reproduced after 4 computation steps and is thus an example of a periodic word.

Clearly, these formal devices are very much in the spirit of the method of combinatory iteration. They really have the appearance of eschewing all interpretation. They are symbol manipulating systems at their purest. But how to study such systems? Post's approach was to start out from the simplest cases, i.e., classes of tag systems with a low number of symbols $\mu$ and a low deletion number $v$, look at some particular such cases and then see where that would get him. ${ }^{5}$ By following this approach he was able to prove that one can decide for any tag system $T$ with $\mu=v=2$ and some word $A \in \Sigma^{*}$ whether or not $T$ will generate or produce $A$, viz., what is now called the reachability problem for tag systems [15]. The proof involved "considerable labor" and Post considered it as the "major success" of his Procter fellowship [21, 362]. ${ }^{6}$ However,

\footnotetext{
${ }^{5}$ This is described in the unpublished document [18] available from the Emil Leon Post papers, American Philosophical Society.

${ }^{6}$ Regretfully the proof was never published. A new proof was recently found and published as $[15]$.
} 
going but one small step beyond this, Post discovered a zoo of tag systems of a "bewildering complexity". He studied several particular examples, writing out productions generated from different initial words, but was not able to get a grip on the "problem of tag" - he simply could not find a way to predict and control the behavior of these apparently simple systems. In fact, even the apparently simple example from above proved "intractable" and is up to today still an open problem. Post concluded that given the difficulties involved with trying to understand (the behavior of) these systems that the general problem of "tag" appeared hopeless, and with it our entire program of the solution of finiteness problems" [21,363]. Post had underestimated the complexities such a "primitive form of mathematics" [21,363] can give rise to. By focusing on the behavior of this form he now clearly understood that simplicity in form does not necessarily imply simplicity in behavior and of the problems defined relative to that form. This insight would lay the foundation of Post's thesis I.

After nine months of research Post turned to two other forms that he had developed: the canonical form $C$ - nowadays known as Post production systems in the context of formal language theory - and the normal form. Systems in normal form, shortly, normal systems are a special class of systems in canonical form $C$. A system in normal form has one initial word (postulate) and a finite set of production rules all of the following form:

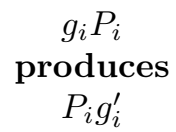

Clearly, normal systems are very similar to tag systems. In fact, the normal form was inspired by the form of "tag" (See [14]).

Knowing from his experience with tag systems that apparent formal simplicity does not necessarily imply real simplicity, Post started on a project of proving the "power" of systems in normal form, viz. their generality: he first proved that canonical form $A$ can be reduced to a system in canonical form $C$ and then, most importantly, proved that the canonical form $C$ reduces to normal form. This fundamental result known as the normal form theorem and once described as "the most beautiful in mathematics" by Marvin Minsky, was later published as [20]. From this Post concluded that in fact the whole of Russel's and Whitehead's Principia Mathematica could be reduced to the normal form:

$[\mathrm{F}]$ or if the meager formal apparatus of our final normal systems can wipe out all of the additional vastly greater complexities of the canonical form [...], the more complicated machinery of [the canonical form] should clearly be able to handle formulations correspondingly more complicated than itself.

It was this additional insight that resulted in the formulation of Post's thesis I, stating that anything that can be "generated" can also be "generated" by the "primitive" normal form. Post next proved that the finiteness problem 
for normal form is unsolvable (incomputable) and, on the assumption of his thesis, concluded that it is unsolvable by any means and, thus, also absolutely unsolvable.

\subsection{Post's thesis II: Solvability and the realm of the com- putable}

When Post became convinced of the universality of his normal form and the absolute unsolvability that comes with it, he was very much aware that he was only able to come to this conclusion because of his own interactions and experiences with this and other forms and that, hence, the thesis would not be so convincing to others who had not had this experience. Thus Post's thesis I is comparable to Church's thesis in the sense that both transpired only after a thorough study of systems of symbolic logic, and, because of that reason, the identifications they propose are not intuitively appealing. Post concluded that "for [the thesis to obtain its] full generality] a complete analysis [should] be made of all the possible ways the human mind [can] set up finite processes for generating sequences." [21, 387].

Not unlike Turing (see the quote on p. 3), Post now set himself the goal of finding a formalism on the basis of an analysis of all the processes one can set up to generate a set, processes which are essentially human processes, processes of the human mind. For that reason [21, 394]:

"[e]stablishing this universality [of the characterization of generated set of sequences in terms of normal form] is not a matter for mathematical proof, but of psychological analysis of the mental processes involved in combinatory mathematical processes.

Only 15 years later Post would publish what is most probably the result of this analysis started in 1921 and interrupted by episodes of his manic-depressive illness and a heavy teaching load (16 contact hours!) at New York City College. ${ }^{7}$ The direct reason for publication was that he had read Church's [1] and was not satisfied with Church's thesis, probably for the same reasons for not being satisfied with his own thesis I. Hence, Post proposed his identification between solvability and solvability by formulation 1 .

Instead of a tape, Post's 'machine' consists of a two-way infinite symbol space divided into boxes. The idea is that a worker is working in this symbol space, being capable of a set of five primitive acts $\left(\mathrm{O}_{1}\right.$ mark a box, $\mathrm{O}_{2}$ unmark a box, $\mathrm{O}_{3}$ move one box to the left, $\mathrm{O}_{4}$ move one box to the right, $\mathrm{O}_{5}$ determining whether the box he is in is marked or unmarked), following a finite set of directions $d_{1}, \ldots, d_{n}$ where each direction $d_{i}$ always has one of the following forms:

(A) Perform one of the operations $\left(\mathrm{O}_{1}-\mathrm{O}_{4}\right)$ and go to instruction $d_{j}$

(B) Perform operation $O_{5}$ and according as the box the worker is in is marked or unmarked follow direction $d_{j^{\prime}}$ or $d_{j^{\prime \prime}}$.

(C) Stop.

\footnotetext{
${ }^{7}$ See [6] for a description of Post's (way of) teaching at City College
} 
Post also defined a specific terminology for his formulation 1 in order to define the solvability of a problem in terms of his formulation 1 . These notions are applicability, finite-1-process, 1-solution and 1-given. Roughly speaking these notions assure that a decision problem is solvable with formulation 1 on the condition that the solution given in the formalism always terminates with a correct solution. As is clear from this description, Post's formulation 1 is almost identical to Turing machines even though there are some interesting smaller differences. $^{8}$

Echoing the reason why he was not fully satisfied with his own thesis I, he emphasized that the purpose of this formulation "is not only to present a system of a certain logical potency but also, [...] of psychological fidelity" [19, 105] Viz., his proposal is not only about defining the scope of the solvable but to capture all possible processes we humans can set-up to solve problems. In this sense, the identification he proposes goes beyond that of the mathematical definition and becomes a "working hypothesis". This is the point at which Post strongly opposes Church, because [19, 105]:

to mask this identification under a definition hides the fact that a fundamental discovery in the limitations of the mathematicizing power of Homo Sapiens has been made and blinds us to the need of its continual verification.

Because of this hypothetical character, Post proposes a program of considering wider and wider formulations and to prove that all these are logically reducible to the original formulation 1 , hence 'testing' the generality of formulation 1 by confronting it with more general formulations. It is the success of such program which, for Post, could change this hypothesis into a "natural law" [19, 105] rather than a definition. ${ }^{9}$

\section{3 "When the bubble of symbolic logic finally burst..."}

Post proposed two logically equivalent but still very different theses, one in 1921 (published in 1943 [20]), and one in 1936. The first thesis was motivated by the insight that a simple form can be much more powerful than its apparent simplicity would suggest. This insight is intimately related to another one: the confrontation with the limitations of symbolic logic. Indeed, it was the experience with his tag systems that led Post to the conclusion that there are limits to what can be achieved with symbolic logic - there are problems that cannot be solved with this "instrument". Even the "primitive" form of "tag" escapes the methods of symbolic logic - a fact unknown to Post but believed and hoped to be true. ${ }^{10}$

\footnotetext{
${ }^{8}$ Like the fact that Post explicitly introduced a halting order.

${ }^{9}$ In this sense, Post believed that the proof of the equivalence between general recursive functions and $\lambda$-definability carries Church's thesis already "beyond the working hypothesis stage" [19, 105].

${ }^{10}$ In one of his letters to Church (dated May 30, 1936) Post expresses this hope by stating that "should [the probem of "tag"] too prove unsolveable I will be supplied with the perfect
} 
The implication of this was clear: if Post's thesis was (and is) true, then the ultimate human method for solving/deciding all mathematical problems in finite time does not exist. Hence, Post 's thesis I, or at least the research from which it originated, is at least as much about the limits of symbolic logic as it is about finding the most general form of (systems of) symbolic logic, characterized as instruments to generate sequences of letters. As such, it emphasizes its own double-faced character, a positive and a negative side (provided one finds the existence of a limitation negative rather than liberating).

Post's second thesis is, in a certain sense, much more about this positive side: its motivation was to somehow capture all the possible processes the human mind can set up to solve decision problems. Thus, Post proposed his program of finding wider and wider formulations, starting from formulation 1, in order to see how far it reaches, to understand what it comprises - how general it actually is, hence effectively extending (our knowledge of) the scope of the humanly solvable/computable. The motivation however for such program lies in the negative side of the thesis. Since Post insisted that his thesis, or any other logically equivalent such thesis, is about human limitations, he found it necessary to explore this limit by studying how far it can be extended.

Post's thesis II identifies solvability in the intuitive sense with solvability by formulation 1. It is oriented towards the idea of defining a formal procedure which somehow captures all processes humans can set up to solve a problem in a finite number of steps. It is about solvability, just as Turing's thesis is about computability and Church's about calculability - notions that are focused on the property that certain "objects" - numbers, functions, problems - are (Turing-) computable, (Church-)calculable or (Post-)solvable. This thesis is concentrated on the idea of having a correct output after a finite number of steps, of effectively being able to have a solution at some point in time. Post's finite-1-processes are supposed to halt on an input, just as Turing machines are in the modern context. This is one of the reasons why (halting) Turing machines are, from a practical (!) point of view, not always considered the most suitable models in certain modern computational situations where, for instance, computer networks are not supposed to halt if they have "solved" one problem. Imagine that your OS would stop working every time you have googled something! In such contexts, alternative models like non-haling Turing machines are more suitable. ${ }^{11}$

Post's "older" models did not have this requirement of termination. This is not surprising knowing how and why these "models" came into existence. They were not intended as models of computability or solvability but rather as instruments of generalization to tackle general mathematical problems like the

alibie for a year of frustration. In a postcard to Church (dated May 3, 1943) he speaks about the "probably unsolveable tag-problem". The letters from Post to Church can be found in the Alonzo Church papers, box 20, Folder 14; Department of Rare Books and Special Collections, Princeton University Library. The problem of "tag" was finally proved unsolvable by Marvin Minsky in 1961 after the problem was suggested to him by Martin Davis [13].

${ }^{11}$ Of course, one should put things in their proper historical context: when Post was proposing his formulation 1 the issue was to find a formulation that captures the vague notion of solvability making it possible to differ between solvable and unsolvable problems. Hence, termination when a solution is found is a very reasonable assumption for the model. 
Entscheidungsproblem, viz., they were intended as general forms that "eschew all interpretation." It is exactly because of this that the form of "tag" can hardly be considered as a good model of computability. However, it is also because of this that the approach Post chose for researching tag systems is one that brings to the foreground not some computable object, but the computational process itself. What matters then is to know what kinds of general properties one can expect from, for instance, the processes induced by the formal rules of the example on p. 6. These tag systems only became a frustration to Post because they take and need their time to develop their behavior and was confronted with the fact that one cannot reach beyond a certain "time horizon". The only way, for Post, to know what would happen even to the simple example of p. 6 was to write down and trace the process induced by the formal definition of that example. As such, the 'time sensitivity' of these computational devices was clear from the start - Post did not need to wait for a computer to understand this.

This focus on the behavior of the computational process itself rather than the 'algorithm' that induces it, results in an approach which is much more based on observation and, in this way, opens up the way for exploratory experimentation, a possibility only made truly feasible with the rise of the electronic and programmable computer. It furthermore introduces a way to think about the borderline between undecidability and decidability in relation to the complexity of the behavior of a given computational process. In this way, one of Post's historically older and less intuitively appealing 'models' are, in a sense, more adept to modern research with its focus on the relation between processes in nature and computation, exactly because of the focus on the computational process itself that tag systems necessitate. ${ }^{12}$

The fact that Post concentrated on studying the behavior of tag systems confronted him with the possibility that his entire program of proving the decidability of the Entscheidungsproblem might be in vain. By exploring the behavior of tag systems he was confronted with his own all too human limitations. ${ }^{13}$ It is exactly this human experience that can help to understand Post's insistence on calling his theses and any other logically equivalent one a hypothesis rather than a definition.

But this view also has a deeper philosophical underpinning. It is rooted in a strong view on the nature of symbolic logic and mathematics, a view which is not completely unrelated to Brouwer's views on the nature of mathematics, to

\footnotetext{
${ }^{12}$ I do not mean that such approach is not possible in the context of, for instance, Turing machines, but that, seen from a historical perspective, tag systems make such approach more apparent.

${ }^{13}$ This "confrontation" went so far that Post reports in one of his letters to Church, dated March 3, 1943, that it was while working on tag systems that he had his first manic-depressive attack: "my wife is much worried. So I told her, really for the first time, the exact history of my mental ups and downs and worse from its first inception in trying to solve the probably unsolvable tag-problem in Princeton" Whether or not this is really true can perhaps be doubted, but it says something about the psychological impact these systems have had on Post, systems he never wanted to work on again (personal communication with Martin Davis).
} 
whose work Post sometimes referred. This view is made explicit here [21, 403]:

I consider mathematics as a product of the human mind, not as absolute

A similar view is expressed when Post explains that the finitary character of symbolic logic follows from the fact that it is "essentially a human enterprise, and that when this is departed from, it is then incumbent on such a writer to add a qualifying "non-finitary"." [21, 394]

Since Post considered mathematics as a product of the human mind, it is understandable that he rejected the idea of the thesis as being but a definition because, from that perspective, you can indeed not "hide behind merely a definition [Post in a letter to Church, dated July 10, 1936] - one cannot isolate the thesis as a thesis about mathematics from the real world mathematician since the only mathematics we know is human mathematics. Hence, if the thesis is correct we are indeed confronted with an absolutely unsolvable problem, viz. unsolvable by the mathematics of humans [21, 340]:

The writer cannot overemphasize the fundamental importance to mathematics of the existence of absolutely unsolvable combinatory problems. True, with a specific criterion of solvability under consideration, say recursiveness [...], the unsolvability in question, as in the case of the famous problems of antiquity, becomes merely unsolvability by a given set of instruments. [The] fundamental new thing is that for the combinatory problems the given set of instruments is in effect the only humanly possible set.

The idea of this absoluteness greatly impressed Post and it is for that reason that he kept insisting on the hypothetical character of his thesis. However, Post understood that this absoluteness is only an absoluteness relative to humans and so "the troubling thought [is suggested] have we so fathomed all our own powers as to insure our assertion of absolute unsolveability relative to us." (Post in a letter to Church, dated July 10, 1936) In the correspondence with Church, Post is clearly searching for ways to "test" the hypothesis. Among others, he considers wider and wider formulations of the Turing machine model. However, it is also clear from that same correspondence that even though the "thesis" should be treated as a hypothesis, Post was strongly convinced of the truth of the hypothesis. To put it in his words (Post in a letter to Church, dated June 9, 1937):

I - to put it crudely but forcibly - am willing to bet a 1000 to $\$ 1$ that [there are no effectively calculable functions that are not recursively solvable], and it would take me five years to save up that sum [...] But I am not willing to stake my "immortal" soul in it - which I should were I to adopt your [Church's] original position [the thesis as a definiton].

Post might have been tempted at that time to conclude that, since we have reached the limitations of (finitary) symbolic logic, we might as well give up on 
it. This was not Post's conclusion: knowing (or believing) that there are things that cannot be achieved with symbolic logic, knowing that there are absolutely unsolvable problems does not mean that one knows what they are or that one knows the realm of the computable. It is here that symbolic logic has a clear future [21]:

with the bubble of symbolic logic as universal logical machine finally burst, a new future dawns for it as the indispensable means for revealing and developing those limitations. For [...] Symbolic Logic may be said to be Mathematics become self-conscious.

Indeed, for Post symbolic logic (or formal logic) can be used as an instrument to explore the limitations exposed through the methods of symbolic logic. ${ }^{14}$

\section{Some afterthoughts}

To expose you, reader, to Post's ideas on computation is the first ambition of this paper but the motivation behind this is not mere historical completeness but rather to add historical and philosophical depth and understanding to the nowadays so fundamental concept of computation and its limitations.

The lessons I learned from Post's writings are manifold, but I think that one of the more important ones lies in his (implicit) insistence on the fact that the thesis is as much about the universe of computation as it is about the limits of that self-same universe, a universe shaped by the possibilities and limitations of human mathematics - why else call it a hypothesis? ${ }^{15}$

Exploring these limitations, this borderline, is in my view still an essential objective in our search for a better understanding of the nature of computation. An 'old' approach to this problem is not to study that which lies "beyond" that borderline, but rather to approximate it, to explore it, by searching for and studying 'smallest' universal and/or undecidable devices and 'largest' decidable classes of such devices. ${ }^{16}$ It is this line of research that allows to excavate this frontier of (Turing-)computability from the bottom up, starting from the computable side of the borderline just up to the edge of that borderline. With the advances in computer hard-and software these excavations are now supported by computer-aided explorations of the behavior of very different classes of computational devices like tag systems [16], Turing machines [12] or cellular automata [3] which, among others, advances our understanding of the computational power of particularly difficult instances like the example on p. 6 .

This kind of research on the limits of (Turing-)computability is perhaps less fashionable today than for instance research on DNA computing, but that does

\footnotetext{
${ }^{14}$ This is exactly what Post partly did in some of his later work: the proof of the unsolvability of the Post correspondence problems and the word problem of semi-groups as well as his fundamental paper on recursively enumerable sets and their decision problems can be understood from this perspective.

${ }^{15}$ Just to be clear, this does not imply some form of computationalism.

${ }^{16}$ See e.g. [11] for a survey of such research.
} 
not mean that it lacks significance. In fact, it is readily seen that such results are paramount to our understanding not only of the nature of computation but also of computation in nature, even more so if we incorporate the computer-aided studies. Indeed, to determine limits of the computable is also to determine the limits of our computational models of physics and biology and the question of what can and cannot be computed in nature goes to the core of what computation in nature means. These kind of results can be achieved by tracing down properties in the behavior of possibly undecidable instances or by learning more about the size of smallest universal devices in computational contexts that are more suited for this kind of research, and then transmuting these findings to the context of natural computing.

Post did not start out from the idea of developing a model that is a suitable model of computability, rather he was searching for the most general form of symbolic logic and ultimately mathematics. This resulted in his apparently simple form of "tag" which lay the basis for his findings on the possibilities and limitations of symbolic logic. Nowadays, models of computability like tag systems seem to be less interesting if one wants to inquire into (the nature of) computation (in nature) because they seem unrelated to nature, because they do not have the "appeal to intuition" that e.g. membrane systems have for cellular behavior. Perhaps however a return to such abstract formal devices that are less 'natural' can rejuvenate or at least stimulate current research on natural computing. Indeed, Post's devices are different enough from physical processes such that they allow to zoom-in on the 'non-physical' aspects of computational processes but are nonetheless erratic, unpredictable and complex in a way that is not completely averse to certain physical processes. In this way, by placing

'nature' between brackets for a while, one can perhaps study the nature of computation by investigating nature taking place in computation.

\section{References}

[1] Alonzo Church, An unsolvable problem of elementary number theory, American Journal of Mathematics (1936), no. 58, 345-363.

[2] — Review of [26], Journal of Symbolic Logic 2 (1937), no. 1, 42-43.

[3] Matthew Cook, Universality in elementary cellular automata, Complex Systems 15 (2004), no. 1, 1-40.

[4] Martin Davis, The undecidable. Basic papers on undecidable propositions, unsolvable problems and computable functions, Raven Press, New York, 1965, Corrected republication (2004), Dover publications, New York.

[5] _ Why Gödel didn't have Church's thesis, Information and Control 54 (1982), 3-24.

[6] _ _ Emil L. Post. His life and work, 1994, in: [22], xi-xviii. 
[7] Liesbeth De Mol, Closing the circle: An analysis of Emil Post's early work., The Bulletin of Symbolic Logic 12 (2006), no. 2, 267-289.

[8] Solvability of the halting and reachability problem for binary 2-tag systems, Fundamenta Informaticae 99 (2010), no. 4, 435-471.

[9] - On the complex behavior of simple tag systems, Theoretical Computer Science 412 (2011), no. 1-2, 97-112.

[10] Robin Gandy, The confluence of ideas in 1936, 1988, Published in [9], pp. 55-111.

[11] Kurt Gödel, Remarks before the princeton bicentennial conference on problems in mathematics, 1946, in: [4], 84-88.

[12] Rolf Herken (ed.), The Universal Turing machine, Oxford University Press, Oxford, 1988, Republication (1994), Springer Verlag, New York.

[13] Stephen C. Kleene, Origins of recursive function theory, Annals of the history of computing $\mathbf{3}$ (1981), 52-67.

[14] Maurice Margenstern, Frontier between decidability and undecidability: A survey, Theoretical Computer Science 231 (2000), no. 2, 217-251.

[15] Pascal Michel, Small Turing machines and generalized Busy Beaver competition, Theoretical Computer Science 326 (2004), no. 1-3, 45-56.

[16] Marvin Minsky, Recursive unsolvability of Post's problem of tag and other topics in the theory of Turing machines, Annals of Mathematics 74 (1961), 437-455.

[17] Emil Leon Post, Introduction to a general theory of elementary propositions., American Journal of Mathematics 43 (1921), 163-185.

[18] Note on a fundamental problem in postulate theory, 1921, dated June 4, 1921, Emil Leon Post papers, American Philosophical Society.

[19] , Finite combinatory processes - Formulation 1, The Journal of Symbolic Logic 1 (1936), no. 3, 103-105, Also published in [4], 289-291.

[20] Formal reductions of the general combinatorial decision problem, American Journal of Mathematics 65 (1943), no. 2, 197-215.

[21] _ Absolutely unsolvable problems and relatively undecidable propositions - Account of an anticipation, 1965, in: [4], 340-433. Also published in [22].

[22] Solvability, Provability, Definability: The collected works of Emil L. Post, Birkhauser, Boston, 1994, edited by Martin Davis.

[23] Wilfried Sieg, Mechanical procedures and mathematical experience, Mathematics and Mind (Oxford), Oxford University Press, 1994, pp. 71-117. 
[24] Robert L. Soare, Computability and recursion, The bulletin of Symbolic Logic 2 (1996), no. 3, 284-321.

[25] _ Computability and incomputability, Computation and Logic in the Real world. Third conference on computability in Europe, CIE2007, Siena, Italy (S.B. Cooper, B. Löwe, and A. Sorbi, eds.), LNCS, Springer, 2007, pp. $705-715$.

[26] Alan M. Turing, On computable numbers with an application to the Entscheidungsproblem, Proceedings of the London Mathematical Society (1936-37), no. 42, 230-265, A correction to the paper was published in the same journal, vol. 43, 1937, 544-546. Both were published in [4], 116-151.

[27] _ Intelligent machinery, a heretical theory, 1951, Lecture given at Manchester (2 versions, one numbered 1-10, the other numbered 96-101). Available at the digital Turing archive at http://www.turingarchive.org, archive number AMT/B/4. 